\title{
SPH smooth particle dynamic method is used to calculate the hypervelocity impact of high temperature ceramic materials
}

\author{
Liu Baoliang $^{\mathrm{a}}$, Zhang Yongke ${ }^{\mathrm{b}}$, Li Qiaoqiaoc
}

\author{
Science college, Heilongjiang University of Science and Technology, Harbin 150022, P.R.China

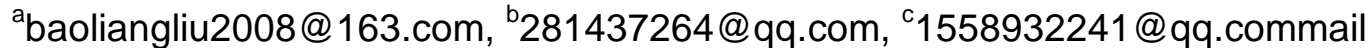

Keywords: Super ultra-high temperature ceramic; Hypervelocity impact; Smooth particle dynamics; Numerical Simulation

Abstract. In this paper, the simulation method is used to study the high-temperature impact of ultra-high temperature ceramic materials with two-stage light-gas cannon projectile.Using ANSYS-AUTODYN dynamic program, smooth particle hydrodynamics and finite element mesh coupling algorithm (SPH-FEM) were used.The numerical simulation of the local perforation behavior of the hypervelocity impacted ultra-high temperature ceramic material plate with spherical projectile was carried out.The faster the projectile speed is, the greater the diameter of the perforations on the ceramic target plate. At the same time, the cracks in the ceramic target plate are less and less; and the debris cloud also has an oval state into a spherical state.

\section{The introduction}

Countries are developed for hypervelocity impact hitting the ground simulation experimental study of various accelerating technology and launchers, using these devices to a large number of simulation experiments on the ground, abundant research results have been achieved[1].There are many kinds of high speed launch equipment, including;first class gunpowder, second-level light gas cannon, three-level light gas gun, electromagnetic accelerator, electrostatic accelerator, directional explosion (explosives) accelerator, cluster accelerator, rail gun, etc[2]. Liu sen, Li yi of northwestern institute of nuclear technology and China's air power research and development center,which are based on the secondary light air cannon to carry out ultra-high speed impact experiments and related research,but they are not systematic and comprehensive[3].Funded by the national defense commission, Harbin Institute of Technology has established experimental equipment that can simulate space debris impact on the shield,And having AUTODYN numerical simulation software, The characteristics of debris cloud are studied by means of experiment and numerical simulation. The lab is now preparing to build a three stage light gas gun capable of firing faster projectiles, at the same time, the AUTODYN software can be developed twice,Using the combination of experiment and numerical simulation, the study is carried out on the dynamic mechanical properties of super-high speed impact, debris cloud modeling and damage prediction and materials.

\section{Theoretical model}

The Johnson-Holmquist constitutive model is a constitutive model of brittle materials in ANSYS-AUTODYN。It's expression is:

$$
\begin{aligned}
& P=K_{1}+K_{2} u^{2}+K_{3} u^{3} \\
& \sigma^{\prime}=(1-D) \sigma_{i}^{\prime}+D \sigma_{f}^{\prime} \\
& D=\sum \frac{\Delta \varepsilon^{p}}{\varepsilon_{f}^{p}}
\end{aligned}
$$




$$
P=K_{1} u, u \leq 0
$$

$\sigma_{i}^{\prime}$ is the dimensionless material of the intact material. $\sigma_{f}^{\prime}$ is equivalent stress; $\varepsilon_{f}^{p}$ is the failure strain;D is the ratio of accumulated plastic strain and failure strain.

Smooth particle hydrodynamic method(SPH) is a grid - free method developed over the past 20 years. The basic idea is to discrete the material of the entire flow field into a series of mass, velocity and energy characteristics, and then evaluating the kernel function through an integral called kernel function[4].Thus, the dynamic quantities of different positions at different moments in flow field are obtained,this is a pure pull particle method.There is no need for a grid and the logic is simple[5].

SPH is a particle algorithm, and there are many different kinds of approximate equations. The approximate equation of SPH particle is:

$$
\begin{aligned}
\frac{d \rho_{i}}{d t} & =m_{i} \sum_{j} u_{i j} \nabla_{i} W_{i j} \\
\frac{d u^{i}}{d t} & =-\sum m_{j}\left(\frac{\sigma_{i}^{\alpha \beta}}{\rho_{i}^{2}}+\prod_{i j}\right) w_{i j, \beta} \\
\frac{d E_{i}}{d t} & =-\sum_{j} m_{j}\left(\mu_{i}^{\alpha}-\mu_{j}^{\alpha}\right)\left(\frac{\sigma_{i}^{\alpha \beta}}{\rho_{i}^{2}}+\frac{1}{2} \Pi_{i j}\right) W_{i j \cdot \beta}+H_{\mathrm{i}}
\end{aligned}
$$

$\rho$ is density; $m$ is particle mass; $\sigma$ is particle stress; $u$ is particle velocity; $E$ is specific internal energy; $H$ is

artificial heat flow; $W$ is kernel function; $i, j$ is particle numbering.

\section{The geometric model}

The secondary light air gun can launch high speed spherical projectiles, conical projectiles, bullet-shaped projectiles and other types of projectiles, The shape of the projectiles is different in the shape and shape of the projectiles, This paper mainly studies the damage status and shape of the target plate and ball projectile when the spherical projectile hits the ceramic target plate, The impact velocity of the projectile is $1000 \mathrm{~m} / \mathrm{s}, 1400 \mathrm{~m} / \mathrm{s}, 1480 \mathrm{~m} / \mathrm{s}, 2200 \mathrm{~m} / \mathrm{s}, 2600 \mathrm{~m} / \mathrm{s}, 3000 \mathrm{~m} / \mathrm{s}$.

As shown in Fig.1, this image is a geometric model of simulated second-stage light gas cannon blasting ceramic plates with spherical projectiles。 The spherical pellet material is AL2024T351 with a radius of $3 \mathrm{~mm}$. The material of the board is $\mathrm{ZrB}_{2}-\mathrm{SIC}$ of the high temperature ceramic material of boride, the height of the plate is $60 \mathrm{~mm}$, the width is $40 \mathrm{~mm}$, the thickness is $1 \mathrm{~mm}, 3 \mathrm{~mm}, 5 \mathrm{~mm}, 7 \mathrm{~mm}$ and $9 \mathrm{~mm}$.

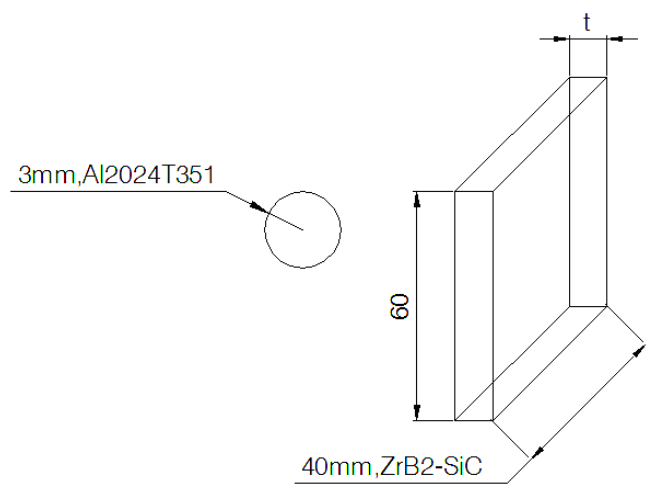

Fig.1 Geometric model 


\section{Numerical simulation}

\section{SPH method}

The main reasons for the simulation of aluminum alloy projectiles and $\mathrm{ZrB}_{2}-\mathrm{SiC}$ ceramic plates without grid SPH are the following:

If aluminum alloy projectile and $\mathrm{ZrB}_{2}-\mathrm{SiC}$ ceramic plate are Lagrang algorithm, When the ceramic plate projectile hits the contact with the projectile, the cells begin to deform to a certain extent, The ANSYS-AUTODYN software usually gets rid of this unit to keep the computation going, and this algorithm is called an invasion algorithm. At this point, the pressure on the front edge of the $\mathrm{ZrB}_{2}-\mathrm{SiC}$ ceramic material will disappear in a short time due to the disappearance of the unit,This situation can directly affect the pressure state in the $\mathrm{ZrB}_{2}-\mathrm{SiC}$ ceramic unit of adjacent position.

In the SPH model, SPH particles will not be removed because of the large deformation of the material.The pressure on the front of the ceramic will remain and change continuously,the strength of the ceramic material will not decrease instantly,this can ensure the accuracy of calculation results.In addition,Ceramics are brittle materials, they are bound to break under impact;Because there is no grid limitation, the SPH model can better represent the movement after the ceramic fragmentation.

\section{Crushing at different speeds}

SPH algorithm is a grid-free algorithm, As shown in the picture, the radius of the projectile is $3 \mathrm{~mm}$,material is Al2024T351,the smallest particle at particle size is $0.25 \mathrm{~mm} 2$. The target plate in the graph is the ultra-high temperature ceramic material $\left(\mathrm{ZrB}_{2}-\mathrm{SiC}\right)$. The length is $60 \mathrm{~mm}$, the width is $40 \mathrm{~mm}$, and the thickness is $1 \mathrm{~mm}$.

The crushing state of the projectile is obvious when the spherical pellets (Al2024T351) pass through the ceramic target plate $\left(\mathrm{ZrB}_{2}-\mathrm{SiC}\right)$. The projectile can produce a large amount of debris cloud after passing through the target plate. Comparing and analyzing by Fig.2, it is found that the debris cloud is ellipsoidal when the velocity of the debris cloud is relatively low, as the speed increases, the state of debris clouds tends to be spherical. This means that the larger the velocity, the bigger the kinetic energy that the debris cloud carries. It is found that the number of cracks on the high temperature ceramic target is changed obviously by observing the impact of the super high temperature ceramic target, the larger the velocity, the smaller the crack on the ceramic target. It can be concluded that the greater the velocity is, the greater the kinetic energy is converted into debris cloud, and the less the internal energy received by the ceramic target.

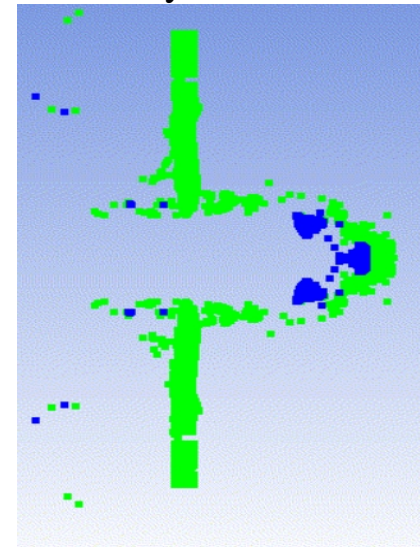

(a) $1000 \mathrm{~m} / \mathrm{s}\left(\mathrm{ZrB}_{2}-\mathrm{SiC}\right)$

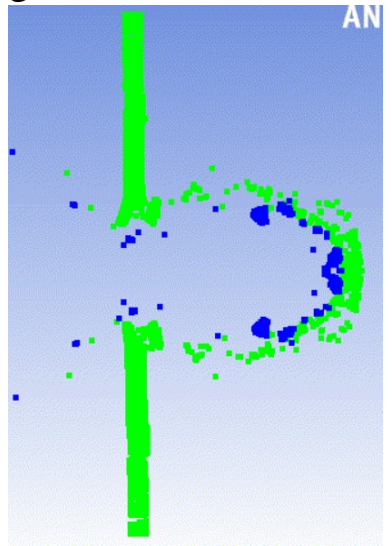

(b) $1400 \mathrm{~m} / \mathrm{s}\left(\mathrm{ZrB}_{2}-\mathrm{SiC}\right)$

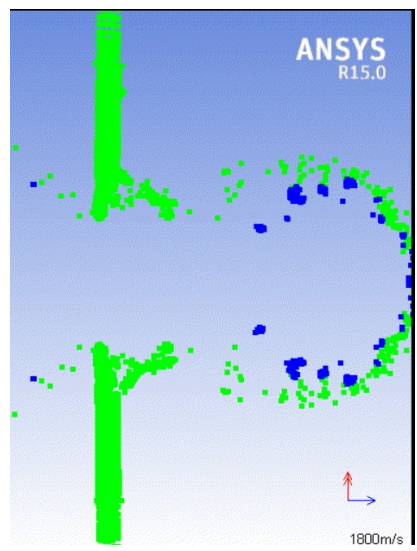

(c) $1800 \mathrm{~m} / \mathrm{s}\left(\mathrm{ZrB}_{2}-\mathrm{SiC}\right)$

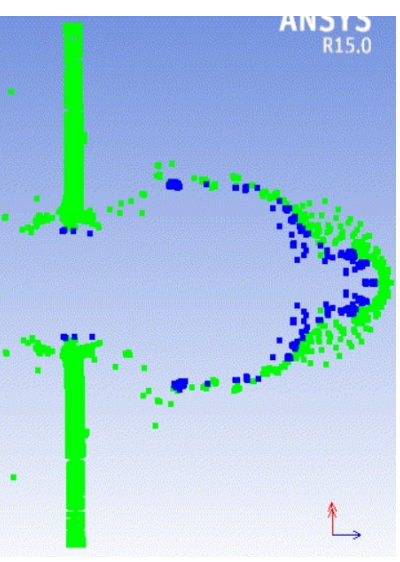

(d) $2200 \mathrm{~m} / \mathrm{s}\left(\mathrm{ZrB}_{2}-\mathrm{SiC}\right)$ 


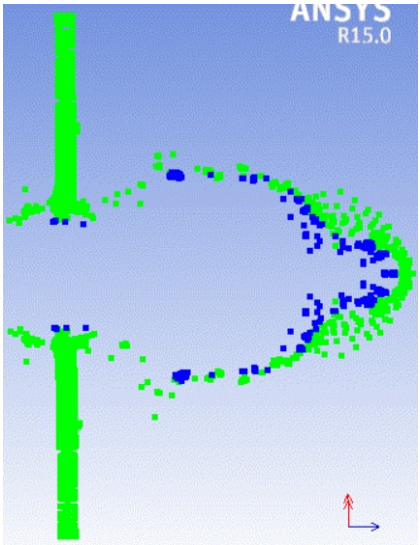

(e) $2600 \mathrm{~m} / \mathrm{s}\left(\mathrm{ZrB}_{2}-\mathrm{SiC}\right)$

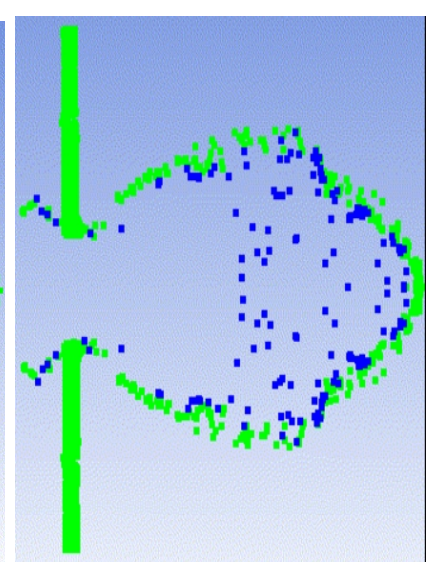

(f) $3000 \mathrm{~m} / \mathrm{s}\left(\mathrm{ZrB}_{2}-\mathrm{SiC}\right)$

Fig.2 Destruction mode under high impact load

Observed the debris cloud, the debris cloud of the super-high temperature ceramic material is relatively loose, the metal materials are relatively concentrated due to their good plasticity.

$$
W=T_{i}+T_{j}+Q
$$

$\mathrm{W}$ is the total kinetic energy, $T_{i}, T_{j}$ are the kinetic energy and the kinetic energy of the pellet debris after impact, $Q$ is the other energy. Through energy analysis, after the impact,the kinetic energy of the ball is transformed into the kinetic energy of the target plate debris and the small debris cloud.In addition,it also exist the internal energy of the plate and the dissipated heat energy. The initial kinetic energy of the ball is $0.15735 \mathrm{~kJ}$, the kinetic energy of the debris cloud of the ceramic target plate after impact is $\mathrm{Ti}=0.06925 \mathrm{KJ}, \mathrm{Tj}=0.0107 \mathrm{KJ}$. The steel target is kinetic energy, $T_{i}$ is $0.0817 \mathrm{~kJ}, T_{j}$ is $0.0269 \mathrm{~kJ}$. Through analysis, the steel target plate can absorb more energy through deformation.

\section{Conclusion}

(1) When the kinetic energy of the aluminum projectile is larger than the penetrating energy of the target plate, the target plate will form a perforation failure.

( 2 ) The radius of projectile velocity increasing damage target is also bigger, and the stress concentration around the crater and the extent of damage is greater.

( 3 ) The kinetic energy of the projectile increases with the increase of velocity, the temperature of the impact is higher,the distribution of temperature fields is also more widespread,so the damage is greater.

( 4 ) As the plate thickened, the damage to the tiles was no longer perforated but broken.The debris cloud formed after the breaking of the ceramic is the spheroid of the elliptic sphere at a small speed, and then as the velocity increases, the cloud of debris tends to be circular.

\section{Acknowledgements}

Special thanks are due to the National Natural Science Foundation of China (No.11572113), Heilongjiang natural science fund project(A2017009) and Harbin City Outstanding Youth Project(2017RAYXJ008).

\section{Reference}

[1]Jinsong Bai, Xiang Wang, Jinsong Hua, et al. The research progress of the numerical simulation of super high speed collider is obtained[J]. Chinese science: Physics Mechanics Astronomy, 2014(05):547-556. 
[2]Tang Qi.High speed impact plate porter and acoustic emission space debris in orbit perception technology[D].Chinese doctoral dissertation.2010.

[3]Yongqiang Zhang.Modeling and damage prediction of debris cloud features of high speed impact protection screen[D].Harbin Institute of Technology.2005.

[4]Jinzhong $\mathrm{Xu}$.The design and study of the high speed collision of space debris and the SPH's method of the protective structure. [D].Doctoral dissertation of China university of science and technology.2010

[5]Jinzhong $\mathrm{Xu}$,Wenhui Tang.SPH simulation of space debris hypervelocity collision problem[J].Intensity and environment.2008,(05):27-33. 